\title{
PHOTOSUBSTITUTION REACTIONS OF AROMATIC COMPOUNDS
}

\author{
J. CORNELISSE \\ Gorlaeus Laboratories, Department of Organic Chemistry, \\ University of Leiden, \\ P.O. Box 75, Leiden, The Netherlands
}

\begin{abstract}
In nucleophilic aromatic photosubstitution, just as in aromatic substitution in the ground state, substituents can have directing and activating effects. Four rules, which describe orientation of nucleophilic substitution in the excited state, can now be formulated. They are:

(a) meta-activation by the nitro group

(b) ortho/para-activation by the methoxy group (and probably also by other electron-donating groups)

(c) ' $\alpha$-reactivity' in bi- and tricyclic aromatics (i.e. position 1 in naphthalenes and azulenes, 9 in phenanthrene, 2 and 4 in biphenyls, etc.)

(d) merging (resonance) stabilization during product formation.

In most nucleophilic aromatic photosubstitutions the reaction proceeds via a $\pi, \pi^{*}$ excited triplet state, which interacts with the nucleophile. In some cases the reaction starts from a $\pi, \pi^{*}$ excited singlet and in some others there are indications that the aromatic molecule in its excited state undergoes dissociation, producing an ion which subsequently reacts with the nucleophile.

Kinetic measurements have revealed the absolute necessity of using rate constants instead of quantum yields as a measure of reactivity. Rate constants for the process in which the triplet excited molecule undergoes deactivation by the nucleophile, leading to substitution product, have been determined for some naphthalene derivatives.

From flash photolytic investigations in the nanosecond region evidence has been obtained for the occurrence of a complex (possibly a sigma-complex) formed from the nucleophile and the aromatic molecule in its reactive excited state. The complex may dissociate back into the starting materials or lead to substitution products, while a third route gives rise to the radical anion. The latter cannot be intermediate in the route to substitution product; instead, it may undergo protonation and be transformed into reduction products.
\end{abstract}

\section{INTRODUCTION}

In this paper a summary is given of our present knowledge on aromatic photosubstitution. The following aspects will be treated: (a) orientation rules; (b) the nature of the reactive excited state and of the primary step; kinetic results; (c) intermediates in aromatic photosubstitution.

The emphasis will lie on nucleophilic aromatic photosubstitution. A 


\section{J. CORNELISSE}

survey of pertinent literature ${ }^{1}$ shows that the number of known nucleophilic photosubstitution reactions far exceeds that of electrophilic photosubstitutions. In this respect the situation differs from that in the ground state. Apparently, the aromatic nucleus becomes very electrophilic upon excitation. As will be seen from the examples below, this is true for unsubstituted as well as for differently substituted aromatics. Many attempts have been made to accomplish electrophilic photosubstitution, but only a few examples have been reported, for instance the photochemical exchange of hydrogen isotopes $2,3,4$ and photo-deboronation ${ }^{5}$. The scarcity of electrophilic aromatic photosubstitutions need not be due to a lack of reactivity of the excited aromatic molecule toward electrophilic reagents; it is known that on excitation the basicity of many aromatic molecules is increased ${ }^{6}$. But many electrophilic reagents cannot be used because they already attack the molecule in its ground state, and many others have been found to very effectively quench the molecule in an excited state.

The paper on aromatic photosubstitution presented to the Second International Symposium on Photochemistry ${ }^{7}$ in 1967 was titled: 'Some problems in aromatic photosubstitution'. We are happy to be able to present answers to some of these problems. But we are equally happy that since that time many new and intriguing problems have been encountered which will stimulate and inspire workers in this field for a long time to come.

\section{ORIENTATION RULES}

Almost simultaneously with the discovery of heterolytic aromatic photosubstitution, it was found that the orientation of substitution in the excited state differs from the well-known rules for the ground state ${ }^{8}$. From the large number of aromatic photosubstitutions found through systematic variation of the type of aromatic nucleus, leaving groups, substituents and the attacking reagent it is now possible to deduce four rules which describe orientation in nucleophilic aromatic photosubstitution ${ }^{1,9}$.

The oldest of these rules is meta-activation by the nitro group. It was first observed in the photosubstitution of nitrophenyl esters and ethers by various nucleophiles ${ }^{8,10}$. Since that time many more examples have been found, demonstrating this meta-directing effect to be a general phenomenon, occurring in different types of nitro-substituted aromatic compounds. Most of these reactions have been reported and described in recent literature (cf. ref. 1); for the present discussion it seems sufficient to mention just a few representative examples.

The behaviour of 4-nitroveratrole is illustrative, because it clearly demonstrates the difference in orientation of substitution in ground state and excited state ( Figure 1).

Thermal hydrolysis, at about $80^{\circ} \mathrm{C}$ in alkaline medium, yields 2-methoxy4-nitrophenol, whereas photochemically at room temperature substitution takes place at the carbon atom meta to the nitro group, giving rise to 2-methoxy-5-nitrophenol in high yield (80 per cent) ${ }^{11}$.

In 2,3-dimethoxy-5- and -6-nitronaphthalene (Figure 2) the activating and directing effect of the nitro group is seen to be effectively transmitted through the ring system in the excited state. In the 5-nitro isomer the methoxy 

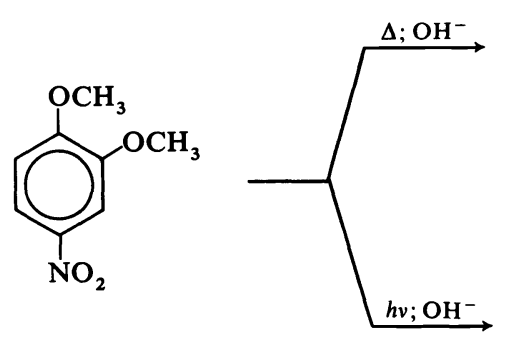<smiles>COc1cc([N+](=O)[O-])ccc1O</smiles><smiles>COc1ccc([N+](=O)[O-])cc1O</smiles>

Figure 1. Thermal and photochemical hydrolysis of 4-nitroveratrole ${ }^{11}$.<smiles>COc1cc2c([N+](=O)[O-])cccc2cc1ONC(C)C</smiles><smiles>COc1cc2ccc([N+](=O)[O-])cc2cc1O</smiles>

Figure 2. Photohydrolysis of 2,3-dimethoxy-5- and -6-nitronaphthalene ${ }^{12}$.

group at carbon atom 2 is displaced by hydroxide ion, while 2,3-dimethoxy6-nitronaphthalene undergoes photosubstitution at position $3^{12}$. Both reactions have a quantum yield at $313 \mathrm{~nm}$ of about 0.10 (at $0.14 \mathrm{M} \mathrm{OH}^{-}$).

If the ortho/meta/para nomenclature is extended to derivatives of naphthalene, it can be seen that in both these reactions the substitution occurs at a position which can be designated as meta to the nitro group.

The change in orientation as caused by the nitro group from ortho/para in the ground state to meta in the excited state is curious and intriguing, but the fact that this group is still an activator for nucleophilic photosubstitutions in the excited state is much less unexpected. Therefore it came as a surprise to find that the methoxy group (and probably also other electron-donating substituents) not only has a directing effect in aromatic photosubstitution, which is again opposite to that expected for the ground state, but also an activating influence on nucleophilic attack.

Figure 3 shows the reactions of anisole and para-chloroanisole with cyanide ion.

The photocyanation of anisole was reported by Nilsson ${ }^{13}$ who found that 


\section{J. CORNELISSE}<smiles>COc1ccc(C#N)cc1</smiles><smiles>COc1ccc(C#N)cc1</smiles>

Figure 3. Photocyanation of anisole ${ }^{13}$ and para-chloroanisole ${ }^{15}$.

the ortho/para ratio of substitution is about 50:50, while the amount of meta-product is negligible. This reaction needs oxygen (or possibly other oxidants). Earlier it was found by Barltrop and co-workers ${ }^{14}$ that in a nitrogen atmosphere a small yield (three per cent) of benzonitrile is obtained by irradiation of anisole with cyanide ion in methanol.

The reaction of para-chloroanisole with cyanide ion proceeds very smoothly ${ }^{15}$. Quantum yield studies are presently being performed and preliminary measurements indicate that at $0.1 \mathrm{M} \mathrm{KCN}$ the quantum yield of formation of para-cyanoanisole may be as high as 0.5 . The same is true for the corresponding fluoro compound.

We have investigated the photocyanation of the three isomeric dimethoxybenzenes. The results are shown in Figure 4.

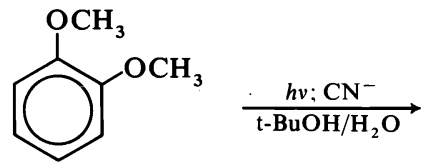<smiles>COc1ccccc1C#N</smiles><smiles>COc1cccc(OC)c1</smiles><smiles>COc1ccc(C#N)c(OC)c1</smiles><smiles>COc1ccc(OC)cc1</smiles>

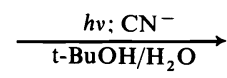<smiles>COc1ccc(C#N)cc1</smiles>

Figure 4. Photocyanation of the three dimethoxybenzenes ${ }^{15}$. 


\section{PHOTOSUBSTITUTION REACTIONS OF AROMATIC COMPOUNDS}

Product yields (based on the amount of starting material consumed) are as follows: ortho 68 per cent; meta 48 per cent; para 21 per cent. In these three compounds photosubstitution occurs at positions which are ortho or para (or both) with respect to methoxy groups. Most interesting is the case of 1,3-dimethoxybenzene where a hydrogen atom (ortho and para to methoxy) is substituted in preference to the better leaving group methoxyl.

The results for the trimethoxybenzenes are entirely similar ${ }^{15,16}$. They are shown in Figure 5.<smiles>COc1cc(OC)cc(OC)c1</smiles>

Figure 5. Photocyanation of the three trimethoxybenzenes ${ }^{15.16}$.

Again, as in the reaction of the 1,3,5-isomer found by Lok and Havinga ${ }^{16}$, if no methoxy group is present which is ortho and/or para to one of the others, a hydrogen atom is substituted.

In the reactions of methoxy-substituted compounds shown thus far, cyanide ion has been used as a nucleophile. As with nucleophilic photosubstitution in the ground state, cyanide is one of the most effective nucleophiles for displacing hydrogen. That nucleophilic photosubstitution in derivatives of anisole can also be effected with other reagents is shown by the example given in Figure 6. High yields of substitution product are obtained upon irradiation of para-chloroanisole in the presence of hydroxide or methoxide ions ${ }^{15}$. In some cases methoxide ion can displace hydrogen ${ }^{17}$, though cyanide is far more effective in this respect.

It should be pointed out that benzene does not give detectable amounts of benzonitrile upon irradiation in the presence of cyanide ion ${ }^{18}$, while chlorobenzene is also very unreactive ${ }^{15}$. There are no indications that introduction of a methoxy group causes an increase in the lifetimes of the excited states (singlet or triplet) that would explain the high yield of substituted product. Therefore one has to assume that the methoxy group really has an activating effect on nucleophilic photosubstitution. 


\section{J. CORNELISSE}

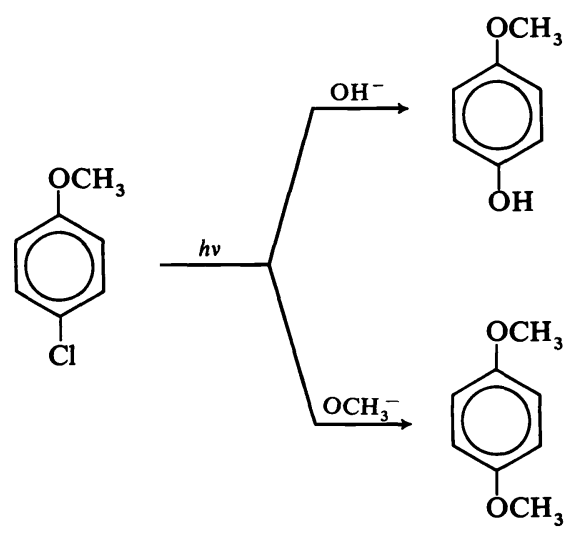

Figure 6. Photoreaction of para-chloroanisole with hydroxide ion and with methoxide ion ${ }^{15}$.

In methoxy-substituted naphthalenes and biphenyls similar reactions occur. In Figure 7 the behaviour of photo-excited 1- and 2-methoxynaphthalene toward cyanide ion is shown.<smiles>COc1ccc2ccccc2c1</smiles>

Figure 7. Photocyanation of 1-methoxynaphthalene ${ }^{16}$ and 2-methoxynaphthalene $\mathrm{e}^{19}$.

The reaction of 2-methoxynaphthalene has been discovered by Letsinger ${ }^{19}$. In addition to the ortho/para activation by the methoxy group, there may be another effect operating here: a difference in reactivity at the $\alpha$ - and $\beta$ positions in naphthalene. Such a difference has indeed been found and it is an example of our third orientation rule. Before we discuss this rule, however, it is instructive to look at some reactions of dimethoxynitrobenzenes with cyanide ion, shown in Figure 8.

The most interesting of these photoreactions is that of 3,5-dimethoxynitrobenzene. The two products, 2-cyano-3,5-dimethoxynitrobenzene and 3-cyano-5-methoxynitrobenzene, are formed in yields of 27 and 6 per cent, respectively. Thus, the double activation by the methoxy groups (ortho and para) of the position ortho to nitro clearly outweighs the single activation 
PHOTOSUBSTITUTION REACTIONS OF AROMATIC COMPOUNDS<smiles>COc1cc(OC)cc([N+](=O)[O-])c1</smiles><smiles>COc1ccc(OC)c([N+](=O)[O-])c1</smiles>

Figure 8. Photochemical reactions of 3,5-dimethoxy-, 2,5-dimethoxy- and 2,4-dimethoxynitrobenzene with cyanide ion ${ }^{20}$.

of the position meta to nitro and the effect of the presence of a better leaving group at that position.

This orientation rule, ortho/para activation by the methoxy group, may probably be extended to other electron-donating substituents. Examples are to be found in the work of El'tsov and co-workers ${ }^{21,22}$ who reported reactions in which the hydroxy, the amino and dialkylamino groups seem to have an ortho/para activating influence. These authors frequently use nitrite ion to photochemically introduce a nitro group.

The fact that photoexcited benzene does not react with cyanide ion has already been mentioned. Some polycyclic hydrocarbons, however, do undergo photocyanation ${ }^{18}$. The reactions of biphenyl, naphthalene and azulene are shown in Figure 9.
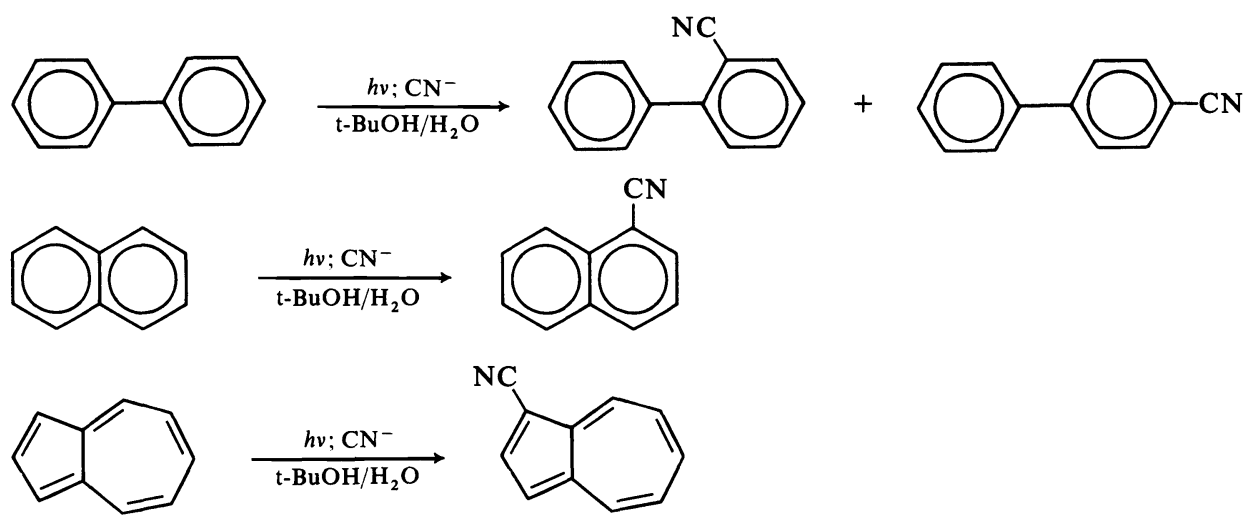

$\frac{h v ; \mathrm{CN}^{-}}{\mathrm{t}-\mathrm{BuOH} / \mathrm{H}_{2} \mathrm{O}}$<smiles>N#Cc1ccc2cccccc1-2</smiles>

Figure 9. Photocyanation of biphenyl, naphthalene and azulene ${ }^{18}$. 


\section{J. CORNELISSE}

In biphenyl and naphthalene the substitution takes place at the positions that are also the most reactive ones in the ground state, for nucleophilic as well as for electrophilic attack. In azulene the situation is different. In the ground state positions 1 and 3 bear the highest negative charge, while carbon atoms 4 and 8 are positively charged ${ }^{23}$. The results of ground state substitution are in accord with this distribution of charge, nucleophilic substitution occurring predominantly at the 4-position and electrophilic reagents attacking at position $1^{24,25}$. In the excited state the situation is reversed. Nucleophilic substitution now occurs at position 1, i.e. again at the position that is to be expected on the basis of calculated charge densities: in the lowest excited singlet as well as in the triplet state positions 1 and 3 bear the highest positive charge ${ }^{23,26}$.

In phenanthrene the reactive positions for nucleophilic photosubstitution are, as expected, 9 and $10^{27}$.

Our third orientation rule may be referred to as ' $\alpha$-reactivity', indicating the reactivity at the $\alpha$-position in naphthalene, position 1 in azulene, 2 and 4 in biphenyl, etc.

Figure 10 shows the photoreactions with cyanide ion of 4- and 3-nitrobiphenyl ${ }^{28}$.

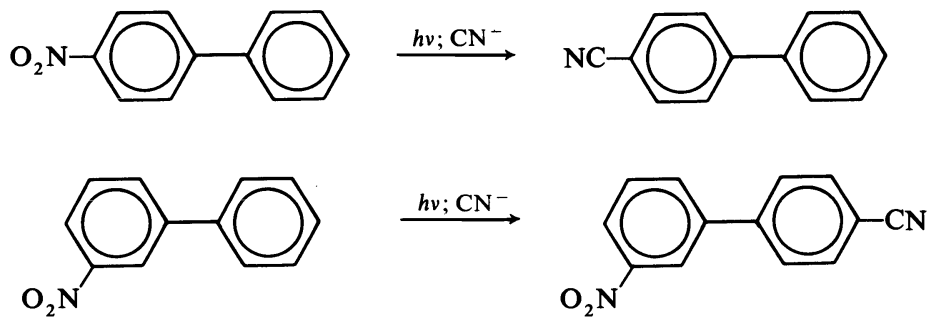

Figure 10. Photocyanation of 4- and 3-nitrobiphenyl ${ }^{28}$.

The result with 3-nitrobiphenyl implies that substitution of hydrogen at the $4^{\prime}$-position is preferred over displacement of a better leaving group at position 3 .

A fourth orientation rule emerges from a study of the effect that the nature of the nucleophile sometimes has on the course of the reaction. As an example, Figure 11 gives two reactions of 1-methoxy-4-nitronaphthalene, one with methylamine leading to $\mathrm{N}$-methyl-1-amino-4-nitronaphthalene, the other, discovered by Letsinger and Hautala ${ }^{29}$, with cyanide ion giving 1-cyano-4-methoxynaphthalene.

The fact that cyanide ion displaces nitrite is not unexpected: here we have the combination of a good leaving group at an $\alpha$-position para to methoxy. But why does the amino group go to the other position? It is also $\alpha$ and there is a good leaving group present, but no activation. In the product, however, we have the combination of a powerful electron-donating substituent and a strong electron-withdrawing group, situated para to each other. From the point of view of resonance stabilization this situation is more attractive than in the product that would be the result of substitution of nitro by amino 

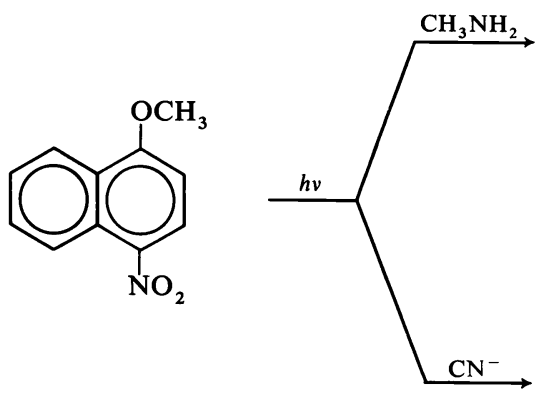<smiles>CNc1ccc([N+](=O)[O-])c2ccccc12</smiles><smiles>COc1ccc(C#N)c2ccccc12</smiles>

Figure 11. Photoreaction of 1-methoxy-4-nitronaphthalene with methylamine and with cyanide ${ }^{29}$.

(or methoxy by cyano). Thus, it seems that the electron-donating or -withdrawing effect that the incoming reagent will have when it becomes fully attached to the aromatic ring already makes itself felt during the product forming steps.

Figure 12 recapitulates some of the reactions of para-nitroanisole with various nucleophiles.
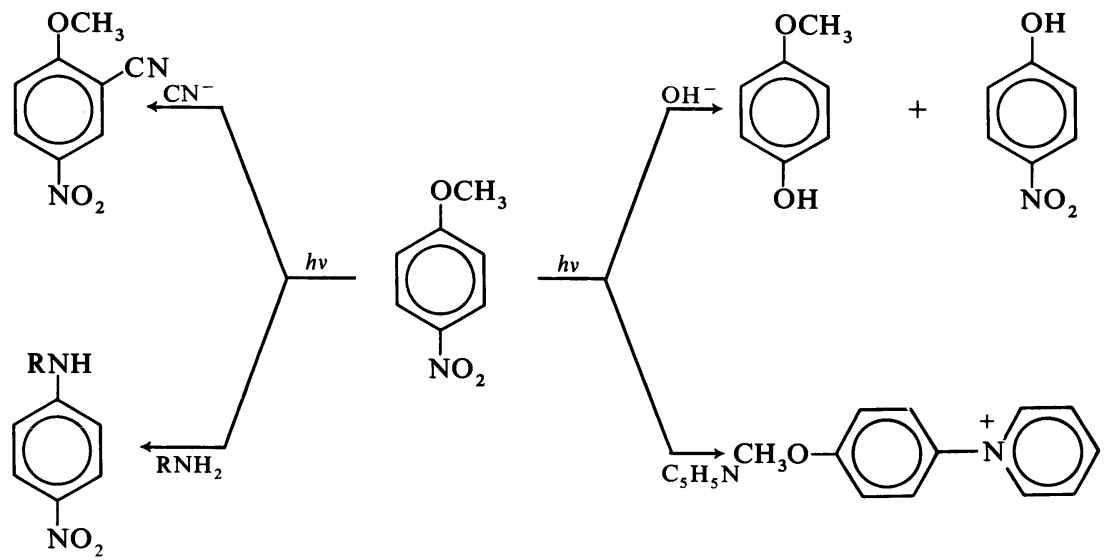

$\mathrm{NO}_{2}^{-}$

Figure 12. Photoreaction of para-nitroanisole with hydroxide $\operatorname{ion}^{30,31}$,yanide $^{300^{32}}$, pyridine $^{30}$, and amines ${ }^{33}$.

On the basis of the orientation rules the position meta to nitro and ortho to methoxy is expected to be the most highly activated one. Of the four nucleophiles shown here, cyanide ion is the one which can most easily substitute hydrogen. Thus, the formation of 2-cyano-4-nitroanisole can be rationalized, as has been pointed out by Letsinger et al. ${ }^{32}$. In the reaction with hydroxide ion the two products are formed in the ratio $4: 1$, substitution of the nitro group predominating ${ }^{30,31}$. This may be another example of the para-directing 


\section{J. CORNELISSE}

effect of the methoxy group (in meta-nitroanisole substitution of the nitro group is not observed, all substitution reactions found involve displacement of methoxide). The difference in behaviour of excited para-nitroanisole toward amines on the one side and pyridine on the other may at least partly be ascribed to resonance stabilization factors. In both cases the product has an electron-donating and an electron-withdrawing group in para-position. To indicate that stabilization of the product already has an effect during its formation, we refer to this phenomenon as 'merging (resonance) stabilization'. This effect also helps to explain the observation that cyanide ion and nitrite ion are the most effective nucleophiles found thus far in photosubstitution of methoxy- and amino-substituted aromatics. This does not mean, however, that this fourth rule can replace the second one: the ortho/para directing effect of methoxy is also found in reactions where the nucleophiles are hydroxide or methoxide ions.

Summarizing, the four orientation rules can be formulated as follows:

(a) meta-activation by the nitro group;

(b) ortho/para activation by methoxy and probably also by other electron donors;

(c) $\alpha$-reactivity in naphthalene and at comparable positions in other bi- and tricyclic aromatics;

(d) 'merging resonance stabilization'.

\section{CHARACTER OF THE EXCITED STATE. KINETICS}

The rules formulated and exemplified in the preceding section have proved to be of predictive value and they can be used in planning syntheses using aromatic photosubstitution. But, like most rules, they have to be applied judiciously. They are still incomplete and may be used only if certain conditions are fulfilled. Relative product yields are of course not only determined by the reactivity of the excited molecule, but also by its lifetime. Besides, much depends on the character of the excited state.

This is nicely illustrated by the behaviour of nitrobenzene in the presence of cyanide ion. On the basis of meta activation by the nitro group and the ability of cyanide to displace hydrogen one might expect to find metanitrobenzonitrile as a photoproduct and one might also be prepared to find some benzonitrile, since nitrite ion is a good leaving group. In fact, as was found almost simultaneously by Petersen and Letsinger ${ }^{34}$ and by Vink et al. $^{35}$, the photoproducts are nitrosobenzene and cyanate ion (Figure 13).

With 1-nitronaphthalene ${ }^{29}, 4$-nitrobipheny $1^{28}$ and 1-nitroazulene ${ }^{36}$ substitution of the nitro group by cyanide ion and other nucleophiles is observed. Figure 14 shows two reactions of 1-nitroazulene, with methoxide and cyanide ion.

A number of photosubstitutions have been found to occur with 1-nitronaphthalene: Figure 15 summarizes some of them.

Obviously and, in view of the distribution of charge, not unexpectedly, a $\pi, \pi^{*}$ excited state rather than an $n, \pi^{*}$ state is required for photosubstitution.

The next question concerns the multiplicity of the excited state. For some photosubstitutions it still has to be established whether they stem from a triplet or from a singlet excited state. In the majority of cases studied thus far a 
PHOTOSUBSTITUTION REACTIONS OF AROMATIC COMPOUNDS

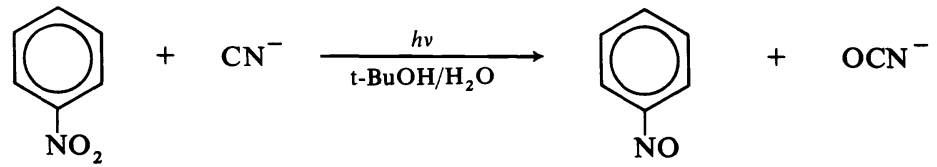

Figure 13. Photoreaction of nitrobenzene with cyanide ion ${ }^{34.35}$.<smiles>O=[N+]([O-])c1ccc2cccccc1-2</smiles>
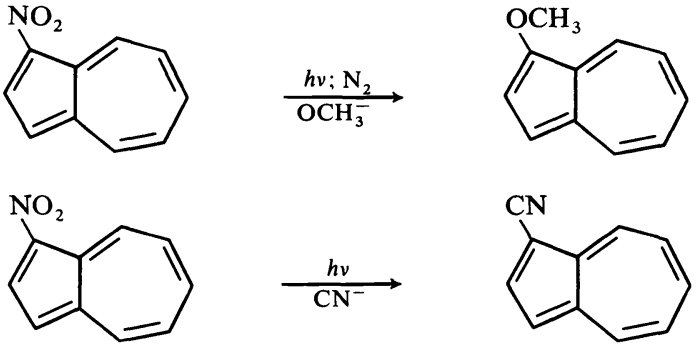

Figure 14. Photoreaction of 1-nitroazulene with methoxide ion and with cyanide ion ${ }^{36}$.

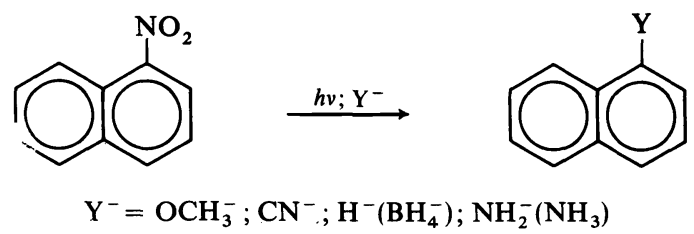

Figure 15. Photoreaction of 1-nitronaphthalene with methoxide ion ${ }^{36}$, cyanide ion ${ }^{29}$, borohydride ion $^{34}$ and ammonia ${ }^{37}$.

triplet state was found to be the reactive intermediate. There do not seem to be any a priori reasons of a structural nature that would make the singlet state a less likely candidate and some singlet reactions have indeed been found. But in these bimolecular processes lifetime is of course a crucial factor.

Until recently most of the aromatic compounds studied in photosubstitution contained a nitro group. Nitroaromatics have two disadvantages in multiplicity studies: they show little or no fluorescence and their triplets have very short lifetimes, usually much too short for discharge tube flash photolysis and in many cases even uncomfortably short for laser flash photolysis in the nanosecond region. We therefore had to resort frequently to indirect methods, mostly sensitization and quenching, with all their inherent dangers of misinterpretation.

Yet, in a number of cases these methods have provided useful evidence concerning the nature of the excited state, together with interesting kinetic results.

In some cases determination of the multiplicity of the reactive excited state may be facilitated by a study of product composition. In the photoreaction of para-nitroanisole with hydroxide ion, Letsinger and Steller ${ }^{38}$ 


\section{J. CORNELISSE}

demonstrated that the ratio of the two products (para-nitrophenol:paramethoxyphenol $=1: 4$ ) is the same in the direct irradiation as in the reaction sensitized by benzophenone.

Irradiation of meta-nitroanisole in liquid ammonia results mainly (more than 90 per cent) in photoamination at the meta-position. In the presence of benzophenone the only product found is 2-methoxy-4-nitroaniline (Figure 16) ${ }^{39}$.<smiles>COc1cccc([N+](=O)[O-])c1</smiles><smiles>COc1cccc([N+](=O)[O-])c1</smiles>

Figure 16. Direct and sensitized photoamination of meta-nitroanisole ${ }^{39}$.

It seems fairly certain that in direct irradiation we are dealing with photosubstitution starting from a singlet excited state.

The photohydrolyses of 2,3-dimethoxy-5- and -6-nitronaphthalene (Figure 2) are also suspected of being singlet reactions ${ }^{12,40}$. Figure 17 shows calculated charge densities for these compounds on positions 2 and 3, obtained from PPP-SCF calculations taking into account configuration interaction of all singly excited singlets ${ }^{41}$.<smiles>O=[N+]([O-])c1cccc2cc(O)c(O)cc12</smiles><smiles>O=[N+]([O-])c1ccc2cc(O)c(O)cc2c1</smiles>

$$
\begin{array}{ccc}
S_{0} & S_{1} & S_{2} \\
+0.001 & +0.053 & +0.021 \\
+0.011 & +0.036 & +0.048 \\
& & \\
& & \\
& & \\
+0.011 & +0.030 & +0.121 \\
0.000 & +0.127 & -0.002
\end{array}
$$

Figure 17. Calculated charge densities on carbon atoms 2 and 3 in 2,3-dimethoxy-5-nitro- and -6 -nitronaphthalene in the ground state and the first and second excited $\pi, \pi^{*}$ singlet state ${ }^{41}$.

In the $S_{1}$ state the highest positive charge is indeed found on the carbon atom meta to nitro, where substitution occurs. We also see that the situation is reversed with respect to the ground state and that the transmission of the 


\section{PHOTOSUBSTITUTION REACTIONS OF AROMATIC COMPOUNDS}

effect of the nitro group is stronger in the excited state than in the ground state, which is also in accord with experimental observations.

In a few cases, for known triplet reactions, charge distributions calculated for the lowest triplet state agree reasonably well with the observed pattern of substitution.

Charge densities in singlet and triplet excited states were also calculated for the various positions in anisole (Figure 18) ${ }^{4}$.
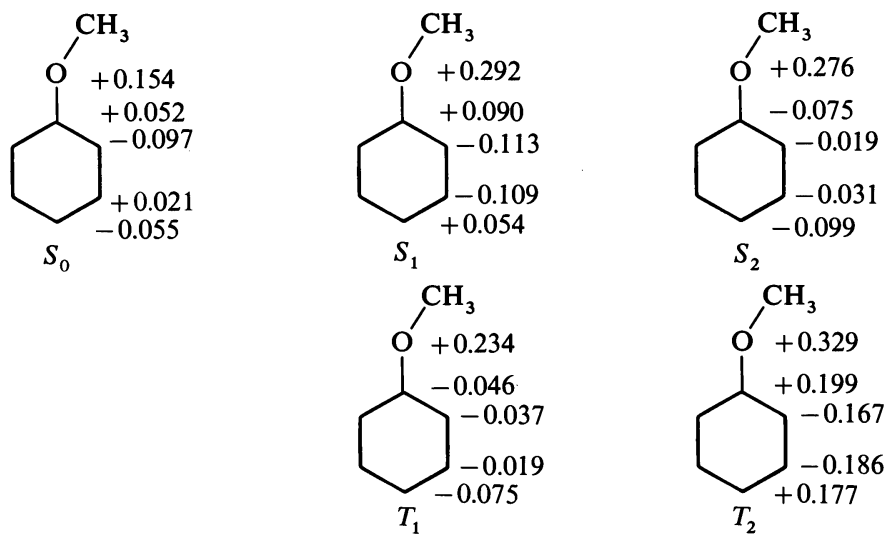

Figure 18. Charge distribution of anisole in the ground state and in singlet and triplet excited states $^{4}$.

Although there are indications that the photosubstitution reactions of anisole and its derivatives may proceed via a triplet state, at the time of writing there is no certainty about this and these recently discovered reactions are still under investigation. It can be seen that in the excited states the methoxy group is still donating electrons to the ring, even to a larger extent than in the ground state. Thus, on the basis of overall charge distribution, no rationalization can be given for the activating effect of methoxy on nucleophilic photosubstitution. In the $S_{1}$ state (and also in $T_{2}$ ) there is at least a positive charge on the para carbon atom, but the ortho activation remains still puzzling.

Summarizing it can be said that although correlations between calculated charge distribution and orientation of substitution have been found in a number of cases, for singlet as well as for triplet reactions, the distribution of charge cannot be the only factor determining orientation. The same is true for localization energies which do not give better results.

It should of course be realized that in using charge distributions in an attempt to rationalize orientation, two assumptions are implicitly made. The first assumption is that the molecule in its excited state interacts directly with the nucleophile and the second one is that the orientation of substitution is determined in that primary step. Both assumptions have to be verified; the first one because the possibility exists that the excited molecule is transformed first into another species (for instance ion or radical) which then interacts with the nucleophile. The second assumption may be unjustified if more than one step is involved in the process leading from excited molecule 


\section{J. CORNELISSE}

to product. In the next section the possible occurrence of intermediates in the photosubstitution reaction will be discussed.

Kinetic investigations have given us useful information regarding the first problem: the nature of the primary step. We have found that most photosubstitutions follow bimolecular kinetics; the rate of product formation depends on the rate of light absorption and on the concentration of nucleophile. The quantum yield of product formation usually equals that of disappearance of starting material and there exists a linear relationship between $1 / \varphi$ and $1 /[\mathrm{N}]$ (where $[\mathrm{N}]=$ nucleophile concentration). With triplet quenchers linear Stern-Volmer plots are found as a rule and by comparing the Stern-Volmer equations at different nucleophile concentrations it appears that the lifetime of the quenched excited molecule depends on that concentration. These facts taken together imply that, at least for most triplet reactions, the excited state is deactivated by the nucleophile and that this deactivation leads to substitution product and possibly also to ground state starting material.

The simplest scheme accommodating these results would be as follows:

$\begin{array}{llll}\mathrm{A} \rightleftarrows{ }^{1} \mathrm{~A}^{*} \rightarrow{ }^{3} \mathrm{~A} & \varphi_{\text {isc }} & \\ { }^{3} \mathrm{~A}^{*} \rightarrow \mathrm{A} & k_{\mathrm{d}} & \text { (pseudo)unimolecular deactivation } \\ \mathrm{N}+{ }^{3} \mathrm{~A}^{*} \rightarrow \mathrm{P} & k_{1} & \text { deactivation by nucleophile leading to product } \\ \mathrm{N}+{ }^{3} \mathrm{~A}^{*} \rightarrow \mathrm{A} & k_{2} & \text { deactivation leading to starting material } \\ \mathrm{Q}+{ }^{3} \mathrm{~A}^{*} \rightarrow \mathrm{A} & k_{\mathrm{q}} & \text { deactivation by triplet quencher }\end{array}$

For the overall kinetics it makes no difference whether the primary product formed from excited molecule and nucleophile is the end product of the reaction or an intermediate which decays without intervention of the nucleophile. In the latter case, if a fraction $\alpha$ would decay into product the scheme would be as follows:

$$
\begin{array}{rlll}
\mathrm{A} \rightleftarrows{ }^{1} \mathrm{~A}^{*} & \rightarrow{ }^{3} \mathrm{~A}^{*} & \varphi_{\text {isc }} \\
{ }^{3} \mathrm{~A}^{*} & \rightarrow \mathrm{A} & k_{\mathrm{d}} & \text { (pseudo)unimolecular deactivation } \\
\mathrm{N}+{ }^{3} \mathrm{~A}^{*} & \rightarrow \mathrm{I} & k_{\mathrm{a}} & \text { formation of intermediate } \\
\mathrm{N}+{ }^{3} \mathrm{~A}^{*} & \rightarrow \mathrm{A} & k_{\mathrm{b}} & \text { deactivation leading to starting material } \\
\mathrm{Q}+{ }^{3} \mathrm{~A}^{*} & \rightarrow \mathrm{A} & k_{\mathrm{q}} & \text { quenching by triplet quencher } \\
\mathrm{I} & \rightarrow \mathrm{P} & \alpha & \text { fraction of intermediate decaying into product } \\
\mathrm{I} & \rightarrow \mathrm{A} & 1-\alpha \text { fraction of intermediate decaying into starting } \\
\end{array}
$$

In that case we measure $k_{\mathrm{a}} \alpha$ instead of $k_{1}$ and $k_{\mathrm{b}}+k_{\mathrm{a}}(1-\alpha)$ instead of $k_{2}$.

The expression for the quantum yield of product formation according to the first scheme is as follows:

$$
\varphi^{-1}=\varphi_{\text {isc }}^{-1}\left[\frac{k_{1}+k_{2}}{k_{1}}+\frac{k_{\mathrm{d}}}{k_{1}}[\mathrm{~N}]^{-1}+\frac{k_{\mathrm{q}}}{k_{1}} \frac{[\mathrm{Q}]}{[\mathrm{N}]}\right]
$$

As an example a few results will be mentioned here which were obtained with some naphthalene derivatives ${ }^{17}$. Several triplet quenchers have been tried of which 3,3,4,4-tetramethyl-1,2-diazetine-1,2-dioxide (TMDD) proved to be very reliable. It has a triplet energy which lies well below that of our naphthalenes and a singlet energy that is high enough to prevent singlet 
energy transfer. It does not quench the fluorescence of naphthalene derivatives even at high concentrations and it gives no ground state complexing ${ }^{\mathbf{4}}$. Quenching rate constants were found to be within the same order as that of other quenchers (like 9-fluorenone) with different chemical properties. It seems safe to assume that quenching by TMDD is diffusion-controlled. The calculated quenching rate constant (room temperature; $\mathrm{CH}_{3} \mathrm{CN} / \mathrm{H}_{2} \mathrm{O}$ $1: 1)$ according to Debye's formula as modified by Beckett, Osborne and Porter ${ }^{43}$ is $1.2 \times 10^{10} \mathrm{lmol}^{-1} \mathrm{~s}^{-1}$.

Figure 19 gives some experimental results for 1-fluoro-3-nitronaphthalene and 1-methoxy-3-nitronaphthalene in their reactions with hydroxide ion and with methylamine. At $0.1 \mathrm{M}$ the quantum yield for the formation of 3-nitro-1-naphthol from the fluoro-compound is 0.33 at $366 \mathrm{~nm}$; from the methoxy-derivative it is 0.036 .

$$
\begin{aligned}
& \phi^{-1}=\phi_{\mathrm{isc}}^{-1}\left[\frac{k_{1}+k_{2}}{k_{1}}+\frac{k_{\mathrm{d}}}{k_{1}}[\mathrm{~N}]^{-1}+\frac{k_{\mathrm{q}}}{k_{1}} \frac{[\mathrm{Q}]}{[\mathrm{N}]}\right] \\
& \overbrace{\mathrm{NO}_{2}}^{\mathrm{C}}
\end{aligned}
$$

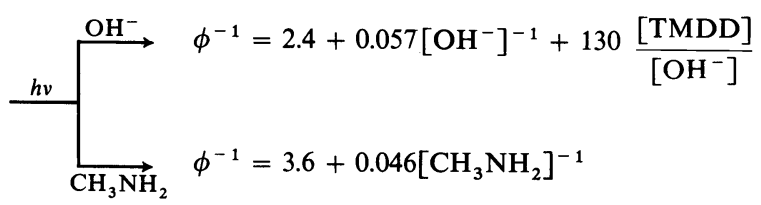

$$
\begin{aligned}
& \overbrace{\mathrm{NO}_{2}}^{\mathrm{OCH}_{3}} \\
& \stackrel{h v}{\underset{\mathrm{CH}_{3} \mathrm{NH}_{2}}{\mathrm{OH}^{-}}} \phi^{-1}=10.0+1.62\left[\mathrm{CH}_{3} \mathrm{NH}_{2}\right]^{-1}+40 \frac{[\mathrm{TMDD}]}{\left[\mathrm{CH}_{3} \mathrm{NH}_{2}\right]}
\end{aligned}
$$

Figure 19. Equations describing the dependence of the quantum yield on the concentrations of nucleophile and quencher for 1-fluoro- and 1-methoxy-3-nitronaphthalene ${ }^{17}$.

The quantum yield of intersystem crossing for 1-fluoro-3-nitronaphthalene has not yet been determined. However, from a study of the absorption and emission spectra of this compound and of 2-nitronaphthalene it appeared that the introduction of a fluoro substituent causes hardly any change in the spectral characteristics. For our calculations we therefore assume the value for the intersystem crossing quantum yield of 1-fluoro-3-nitronaphthalene to be equal to that of 2-nitronaphthalene, viz. 0.8. Introduction of a methoxy group causes appreciable changes in absorption and emission spectra. Thus, for 1-methoxy-3-nitronaphthalene it is not possible to make an accurate guess of $\varphi_{\text {isc }}$ on the basis of a comparison with 2-nitronaphthalene. From the kinetic data a minimum value of 0.1 can be derived. In order to arrive at values for the rate constants which are of the correct order of magnitude, we feel safe in assuming a tentative value of 0.3 for the intersystem crossing quantum yield of 1-methoxy-3-nitronaphthalene. 


\section{J. CORNELISSE}

These values for $\varphi_{\text {isc }}$ together with the diffusion-controlled rate constant for triplet quenching permit us to calculate $k_{1}, k_{2}$ and $k_{\mathrm{d}}$. The results are shown in Table 1. It should be added that the derivation of the expression for the quantum yield rests upon the assumption that $\varphi_{\text {isc }}$ does not depend on the nucleophile concentration. However, if such a dependence would exist there would have been no linear relationship between the reciprocals of quantum yield of product formation and nucleophile concentration, contrary to what has been found.

Table 1. Rate constants for nucleophilic photosubstitution of some naphthalene derivatives ${ }^{17}$

\begin{tabular}{|c|c|c|c|c|}
\hline & $\varphi_{\mathrm{isc}}$ & $\begin{array}{c}k_{1} \\
1 \mathrm{~mol}^{-1} \mathrm{~s}^{-1}\end{array}$ & $\begin{array}{c}k_{2} \\
1 \mathrm{~mol}^{-1} \mathrm{~s}^{-1}\end{array}$ & $\tau=\underset{\mathrm{s}}{1 / k_{\mathrm{d}}}$ \\
\hline 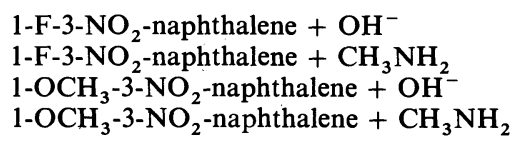 & $\begin{array}{l}0.8 \\
0.8 \\
0.3 ? \\
0.3 ?\end{array}$ & $\begin{array}{l}1.2 \times 10^{8} \\
1.4 \times 10^{8} \\
5.7 \times 10^{8} \\
1.0 \times 10^{9}\end{array}$ & $\begin{array}{l}1.1 \times 10^{8} \\
2.6 \times 10^{8} \\
1.1 \times 10^{9} \\
2.0 \times 10^{9}\end{array}$ & $\begin{array}{l}1.9 \times 10^{-7} \\
1.9 \times 10^{-7} \\
3.2 \times 10^{-9} \\
2.1 \times 10^{-9}\end{array}$ \\
\hline
\end{tabular}

The lifetime of the triplet state, in the absence of quencher and nucleophile, is 60-100 times longer for 1-fluoro-3-nitronaphthalene than for 1-methoxy3-nitronaphthalene. A similar difference has been found at low temperature in rigid solution: the phosphorescence lifetime of the fluoro-compound is an order of magnitude longer than that of the methoxy-derivative. The higher quantum yield of photosubstitution of the fluoro-compound is thus a consequence of the much longer lifetime of the reactive excited state. The reactivity of the molecule in its triplet state, expressed by the value of $k_{1}$, is even higher for the methoxy-derivative. As has been mentioned before, if the reaction should proceed via an intermediate (e.g. a sigma-complex) the value of $k_{1}$ has to be regarded as the product of $k_{\mathrm{a}}$, the rate constant of formation of the intermediate from the molecule in its excited state with the nucleophile, and $\alpha$, the fraction of intermediate decaying into substitution product.

This example illustrates the necessity to use rate constants instead of quantum yields as a true measure of reactivity. In order to arrive at meaningful structure-reactivity relationships many more of these rate constants will have to be determined and their dependence on factors like the type of aromatic nucleus, the leaving group, activating substituents, the attacking reagent, the solvent and the temperature will have to be studied. This is a rather formidable task and we are presently undertaking these studies, using where possible, besides techniques like sensitization and quenching, spectroscopic methods and ultrafast flash photolysis.

Nucleophilic photosubstitution is not limited to carbocyclic aromatic compounds. Heterocyclics like pyridine, thiophene and furan also show this type of reaction. Of the more recent results some reactions of 2-nitrothiophene and 5-bromo-2-nitrothiophene are shown here (Figure 20). ${ }^{44}$ 

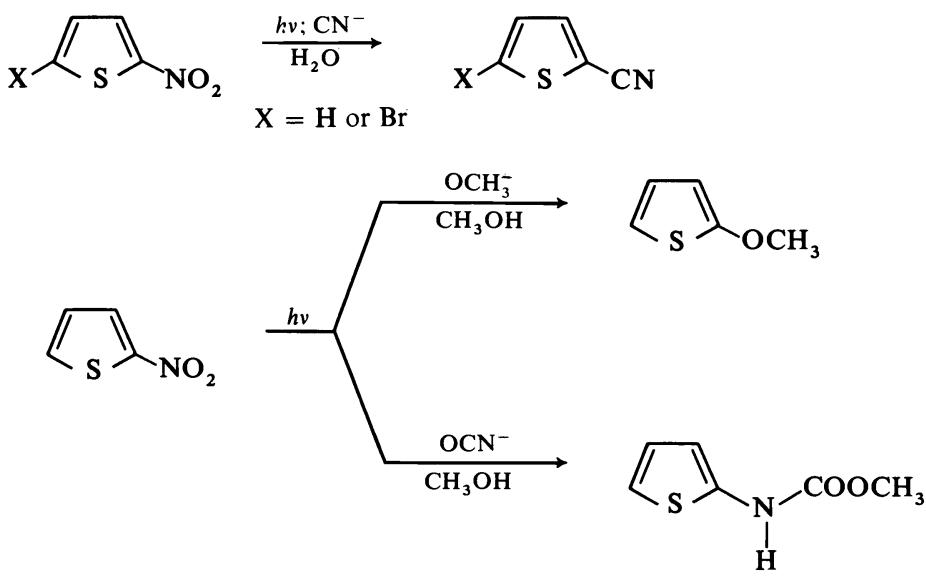

Figure 20. Some photosubstitution reactions of 2-nitrothiophene and 5-bromo-2-nitrothiophene ${ }^{44}$.

Photocyanation of 2-nitrothiophene is a smooth reaction with a quantum yield of $0.39(313 \mathrm{~nm})$ at $0.01 \mathrm{M} \mathrm{KCN}$. The reaction follows the kinetics expected for a bimolecular interaction between excited molecule and nucleophile. The quantum yield of formation of 2-cyanothiophene equals that of disappearance of 2-nitrothiophene. There is the usual linear relationship between the reciprocals of the quantum yield and the nucleophile concentration. The lifetime of the triplet state depends on the amount of nucleophile present: $4.5 \times 10^{-7} \mathrm{~s}$ in water and $1.2 \times 10^{-8} \mathrm{~s}$ in a $10^{-2} \mathrm{M} \mathrm{KCN}$ solution. The 5-bromo-derivative has a considerably higher quantum yield (0.59 at $313 \mathrm{~nm})$.

The photocyanation of 2-nitrofuran is shown in Figure $21 .{ }^{44}$ Here the

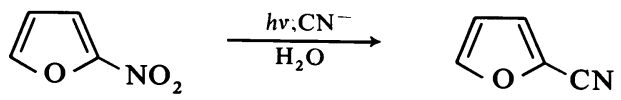

Figure 21. Photocyanation of 2-nitrofuran ${ }^{44}$.

quantum yield of disappearance of the starting material $(0.51$ at $313 \mathrm{~nm})$ is independent of the cyanide ion concentration. The quantum yield of formation of 2-cyanofuran, however, increases with increasing $\mathrm{CN}^{-}$concentration, approaching the value of 0.51 at sufficiently high concentration $(\mathrm{ca} .1 \mathrm{M})$. The formation of 2-hydroxyfuran by reaction with water is thus expected to occur and the presence of its tautomer, $\Delta^{\alpha, \beta}$-butenolide, has indeed been demonstrated.

The photoreaction with cyanide ion can be sensitized and quenched. The triplet lifetime, however, is hardly affected by the presence of cyanide ion, even at a concentration of $0.1 \mathrm{M}$.

To account for the results a reaction scheme is proposed, shown in Figure 


\section{J. CORNELISSE}

22 , where the reactive species undergoing substitution is not the molecule in its triplet state but a species formed from that state. The identity of $\mathrm{X}$ has not yet been established; a positive ion formed in a dissociative process by loss of nitrite ion seems to be an attractive possibility.

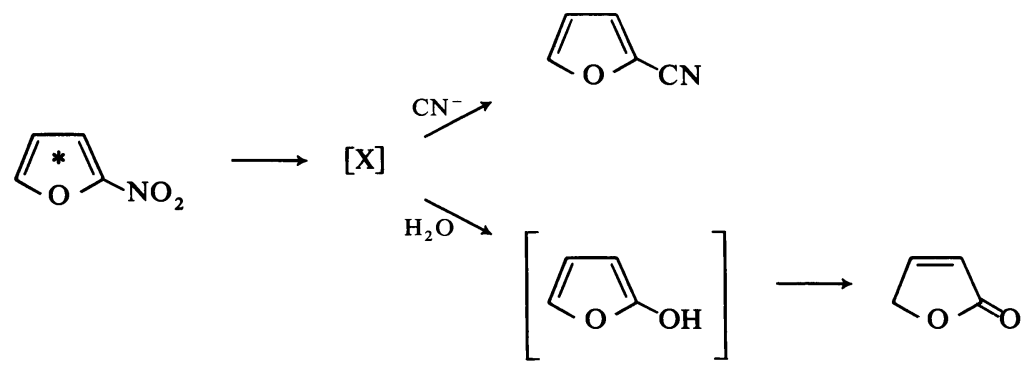

Figure 22. Possible scheme for the photoreaction of 2-nitrofuran with water and with cyanide ion $^{44}$.

In that case we would have an example of an aromatic photosubstitution of tle $S_{N} 1$ type. A similar mechanism has been proposed ${ }^{45}$ for the photohydrolysis of bromopyridines, and it may also be operative in other cases where photosubstitution by water occurs and no increase in the quantum yield is observed in the presence of high concentrations of hydroxide ion ${ }^{46}$.

\section{INTERMEDIATES}

In nucleophilic aromatic substitution in the ground state the existence of sigma-complexes (Meisenheimer-complexes) as intermediates is a welldocumented fact. Most aromatic photosubstitutions hitherto investigated follow bimolecular kinetics. Thus, it seems plausible to assume that these reactions also proceed via an intermediate complex. In the search for intermediates we use conventional (discharge tube) flash photolysis, as well as nanosecond laser photolysis. Especially with the latter technique the investigations are still in their initial stage, but some promising results have already been obtained by De Gunst and Havinga ${ }^{47}$. Only a brief summary of these results will be given here. They concern a flash photolytic investigation of the system 3,5-dinitroanisole + hydroxide ion in acetonitrile-water $(1: 1)$. The product of the photoreaction is 3,5-dinitrophenol, the quantum yield at $313 \mathrm{~nm}: 0.48$.

Three short-lived species have been observed with this system:

(a) A species with an absorption maximum at $550-570 \mathrm{~nm}$ and a lifetime of $40 \mathrm{~ms}$. During the decay of this transient no increase of the extinction at the wavelength of maximum absorption of 3,5-dinitrophenolate ion was observed. The formation of the photoproduct is completed within a much shorter period, even shorter than the flash duration $(20 \mu \mathrm{s})$ of the conventional flash apparatus.

The $40 \mathrm{~ms}$ transient has been identified by means of electron spin resonance as the radical anion of 3,5-dinitroanisole. It can also be generated electrochemically. It is not an intermediate in the photosubstitution reaction, but it 


\section{PHOTOSUBSTITUTION REACTIONS OF AROMATIC COMPOUNDS}

can lead to reduction products via hydrogen abstraction from the solvent.

(b) A species with an absorption maximum at $475 \mathrm{~nm}$ and a lifetime of ca. $12 \mathrm{~ns}$, at $0.04 \mathrm{M} \mathrm{NaOH}$. The same transient is found in neutral solution, but in that medium it has a longer lifetime ( $55 \mathrm{~ns})$. Apparently it is quenched by hydroxide ions. Since the photohydrolysis of 3,5-dinitroanisole is known to be a triplet reaction and the lifetime of the triplet state as calculated by Stern-Volmer experiments is $27 \mathrm{~ns}$, the $475 \mathrm{~nm}$ species may very well be the 3,5-dinitroanisole molecule in its triplet state.

(c) A species with an absorption maximum at $c a .412 \mathrm{~nm}$ and a lifetime of $500 \mathrm{~ns}$. It is rather unfortunate that this is almost exactly the wavelength of maximum absorption of the photoproduct. In this respect 3,5-dinitroanisole has turned out to be a rather poor choice for laser research on intermediates, but of course this was unpredictable. Yet, by carefully monitoring the changes in absorption at different wavelengths it was established by De Gunst and Havinga ${ }^{47}$ that during the decay of the $412 \mathrm{~nm}$ species the absorption in the region of $550 \mathrm{~nm}$ increases. There is an isosbestic point at $535 \mathrm{~nm}$. Very likely the transient at $412 \mathrm{~nm}$ is a precursor of the radical anion. It cannot be detected in neutral solution. Due to the unhappy coincidence of the absorption maxima it could not be established with certainty that the $412 \mathrm{~nm}$ species is a direct precursor of the substitution product. However, as a working hypothesis, we consider this transient to be a complex formed from the aromatic molecule in its triplet state by interaction with hydroxide ion which can decay via three channels: back to ground state starting material, to radical anion and to substitution product.

On the basis of the kinetic and flash photolytic evidence obtained so far, we adopt the reaction scheme presented in Figure 23 as a description of a pathway of aromatic photosubstitution.

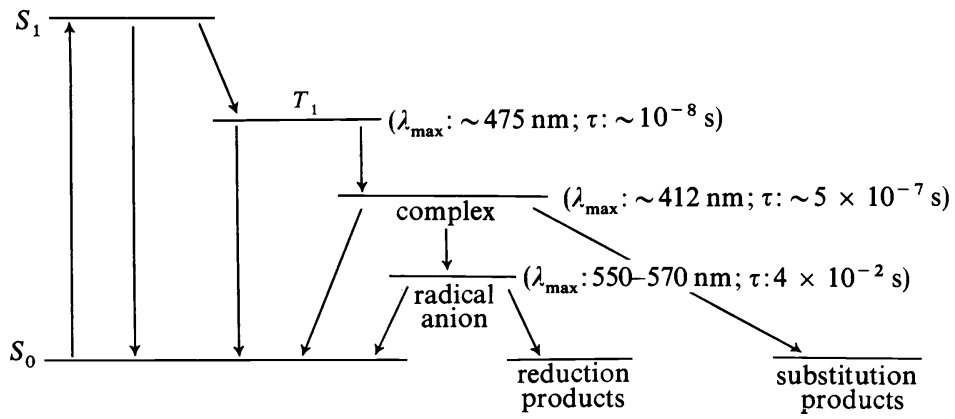

Figure 23. Reaction scheme for nucleophilic aromatic photosubstitution. The values between parentheses refer to the photohydrolysis of 3,5-dinitroanisole in alkaline medium ${ }^{4}$.

\section{ACKNOWLEDGEMENTS}

I wish to express my appreciation to the workers who have carried out the investigations described in this paper: G. M. J. Beijersbergen van Henegouwen, P. A. T. M. Brand, Mrs M. E. den Boer, M. B. Groen, G. P. de Gunst, J. den Heijer, J. G. Lammers, C. M. Lok, G. H. D. van der Stegen, J. J. Tamminga, P. L. Verheijdt, J. A. J. Vink, F. J. Wiegerink. I thank Dr G. 


\section{J. CORNELISSE}

Lodder, Dr J. Lugtenburg and Dr J. J. C. Mulder for valuable discussions and Dr R. A. van Santen and W. T. W. Vis for their assistance with the calculations of charge distributions. Laser experiments were performed at the Laboratory for Physical Chemistry of the University of Amsterdam. The kind help and advice of Professor J. D. W. van Voorst and members of the staff are gratefully acknowledged.

Finally I would like to express my indebtedness to Professor E. Havinga whose invaluable contributions to every aspect in this field of aromatic photochemistry continue to be a source of inspiration. Part of this research was supported by the Netherlands Foundation of Chemical Research (SON) with financial aid from the Netherlands Organization for the Advancement of Pure Research (ZWO).

\section{REFERENCES}

1 J. Cornelisse and E. Havinga, Chem. Rev., to be published.

2 J. P. Colpa, C. MacLean and E. L. Mackor, Tetrahedron, 19, Suppl. 2, 65 (1963).

${ }^{3}$ M. G. Kuz'min, B. M. Uzhinov, G. Szent György and I. V. Berezin, Russ. J. Phys. Chem. 41, 400 (1967).

${ }^{4}$ G. Lodder and E. Havinga, Tetrahedron, 28, 5583 (1972).

${ }^{5}$ F. C. Fischer and E. Havinga, Rec. Trav. Chim. Pays-Bas, 93, 21 (1974).

6 M. G. Kuz'min, B. M. Uzhinov and I. V. Berezin, Russ. J. Phys. Chem. 41, 222 (1967).

${ }^{7}$ E. Havinga and M. E. Kronenberg, Pure Appl. Chem. 16, 137 (1968).

8 E. Havinga, R. O. de Jongh and W. Dorst, Rec. Trav. Chim. Pays-Bas, 75, 378 (1956).

9 J. Cornelisse, G. P. de Gunst and E. Havinga, Adv. Phys. Org. Chem. to be published.

10 E. Havinga and R. O. de Jongh, Bull. Soc. Chim. Belg. 71, 803 (1962).

11 J. L. Stratenus, Thesis, Leiden (1966).

12 G. M. J. Beijersbergen van Henegouwen and E. Havinga, Rec. Trav. Chim. Pays-Bas, 89, 907 (1970).

13 S. Nilsson, Acta Chem. Scand. 27, 329 (1973).

14 J. A. Barltrop, N. J. Bunce and A. Thomson, J. Chem. Soc. (C), 1142 (1967).

$15 \mathrm{~J}$. den Heijer, to be published.

16 C. M. Lok and E. Havinga, Proc. Kon. Ned. Akad. Wetenschap. B, 77, 15 (1974).

17 J. G. Lammers, Thesis, Leiden (1974).

18 J. A. J. Vink, C. M. Lok, J. Cornelisse and E. Havinga, Chem. Commun. 710 (1972).

19 R. L. Letsinger, quoted in Eléments de Photochimie Avancée (ed. P. Courtot), p 343. Hermann: Paris (1972).

${ }^{20}$ F. J. Wiegerink, unpublished results.

21 A. V. El'tsov, A. N. Frolov and O. V. Kul'bitskaya, J. Org. Chem. USSR, 6, 1955 (1970).

22 A. V. El'tsov, O. V. Kul'bitskaya and A. N. Frolov, J. Org. Chem. USSR, 8, 78 (1972).

23 R. Pariser, J. Chem. Phys. 25, 1112 (1956).

24 D. H. Reid, W. H. Stafford and J. P. Ward, J. Chem. Soc. 1100 (1958).

25 A. G. Anderson, D. J. Gale, R. N. McDonald, R. G. Anderson and R. C. Rhodes, J. Org. Chem. 29, 1373 (1964).

26 C. M. Lok, M. E. den Boer, J. Cornelisse and E. Havinga, Tetrahedron, 29, 867 (1973).

27 C. M. Lok, Thesis, Leiden (1972).

28 J. A. J. Vink, P. L. Verheijdt, J. Cornelisse and E. Havinga, Tetrahedron, 28, 5081 (1972).

29 R. L. Letsinger and R. R. Hautala, Tetrahedron Letters, 4205 (1969).

30 R. L. Letsinger, O. B. Ramsay and J. H. McCain, J. Amer. Chem. Soc. 87, 2945 (1965).

31 S. de Vries, Thesis, Leiden (1970).

32 R. L. Letsinger and J. H. McCain, J. Amer. Chem. Soc. 91, 6425 (1969).

${ }^{33}$ M. E. Kronenberg, A. van der Heijden and E. Havinga, Rec. Trav. Chim. Pays-Bas, 85. 56 (1966).

34 W. C. Petersen and R. L. Letsinger, Tetrahedron Letters, 2197 (1971).

35 J. A. J. Vink, J. Cornelisse and E. Havinga, Rec. Trav. Chim. Pays-Bas, 90, 1333 (1971).

${ }^{36}$ C. M. Lok, J. Lugtenburg, J. Cornelisse and E. Havinga, Tetrahedron Letters, 4701 (1970). 


\section{PHOTOSUBSTITUTION REACTIONS OF AROMATIC COMPOUNDS}

37 P. A. T. M. Brand and J. Cornelisse, unpublished results.

38 R. L. Letsinger and K. E. Steller, Tetrahedron Letters, 1401 (1969).

39 A. van Vliet, M. E. Kronenberg, J. Cornelisse and E. Havinga, Tetrahedron, 26, 1061 (1970).

40 G. M. J. Beijersbergen van Henegouwen, Thesis, Leiden (1970).

41 J. J. C. Mulder, R. A. van Santen and W. T. W. Vis, published by G. M. J. Beijersbergen and E. Havinga, ref. 12.

42 E. Ullman and P. Singh, J. Amer. Chem. Soc. 94, 5077 (1972).

43 A. Beckett, A. D. Osborne and G. Porter, Trans. Faraday Soc. 60, 873 (1964).

44 M. B. Groen and E. Havinga, Mol. Photochem. 6, 9 (1974).

45 G. H. D. van der Stegen, Thesis, Leiden (1972).

46 R. O. de Jongh and E. Havinga, Rec. Trav. Chim. Pays-Bas, 87, 1327 (1968).

47 G. P. de Gunst and E. Havinga, Tetrahedron, 29, 2167 (1973). 